\title{
Complete temporal characterization of a single photon
}

\author{
Zhongzhong Qin $^{1,2}$, Adarsh S Prasad ${ }^{1}$, Travis Brannan ${ }^{1}$, Andrew MacRae ${ }^{3}$, A Lezama ${ }^{4}$ and AI Lvovsky ${ }^{1,5,6}$ \\ Precise information about the temporal mode of optical states is crucial for optimizing their interaction efficiency between themselves \\ and/or with matter in various quantum communication devices. Here we propose and experimentally demonstrate a method of \\ determining both the real and imaginary components of a single photon's temporal density matrix by measuring the autocorrelation \\ function of the photocurrent from a balanced homodyne detector at multiple local oscillator frequencies. We test our method on single \\ photons heralded from biphotons generated via four-wave mixing in an atomic vapor and obtain excellent agreement with theoretical \\ predictions for several settings.
}

Light: Science \& Applications (2015) 4, e298; doi:10.1038/lsa.2015.71; published online 5 June 2015

Keywords: autocorrelation matrix; polychromatic optical heterodyne tomography; single photon; temporal mode function

\section{INTRODUCTION}

Single photons and single photon qubits are among the foundations of most quantum optical information processing techniques such as cryptography, ${ }^{1}$ teleportation, ${ }^{2}$ repeaters ${ }^{3}$ and computing. ${ }^{4}$ Many of these applications require the photons to have a well-defined, pure modal structure. Possessing precise information about that structure is essential for quantum optical technology.

An approximate guess of a photon's mode can be inferred theoretically from the characteristics of the source, ${ }^{5-9}$ but this information is not always available or reliable. For example, this approach would not work for photons sent in by a remote party in a communication scheme, or for photons from an incompletely characterized mesoscopic source. Therefore, it is important to have a technique for precise characterization of a photon's mode experimentally. While such techniques are relatively well developed for spatial modes, ${ }^{10,11}$ their extension into the temporal domain is challenging.

One approach to studying the temporal structure of the photon would be to look at the photon detection event statistics as a function of time. For example, this approach has been used to study the timing of coherent double Raman scattering from an atomic ensemble. ${ }^{12}$ Further insight into the photon preparation quality can be gained by studying time-dependent photon counting autocorrelation statistics. ${ }^{13}$ However, these techniques provide no information about the phase coherence between different segments of the photon's temporal mode.

Complete information about a photon's temporal properties can be obtained by studying its interference with a classical field. Polycarpou et al. used adaptive waveform shaping of local oscillator (LO) pulses ${ }^{14}$ to heuristically find the LO temporal mode that maximizes the efficiency of homodyne detection of the photon. This occurs when the LO temporal mode matches that of the signal, enabling measurement of that mode. However, physical shaping of LO pulses is quite sophisticated experimentally. Furthermore, this technique has only been demonstrated for pure temporal modes.

An alternative approach to measuring the spectral density matrix of the photon has been proposed in Ref.15. It is based on bringing the photon into interference with a pair of weak coherent pulses with varied separation between them. However, this method can only be applied to ultrashort pulses whose width is a few optical cycles. Furthermore, the experimental test in Ref. 15 has been performed on a thermal state rather than the single photon state.

For a photon with bandwidth resolvable by detection electronics, the time domain statistics may be measured directly and in real time by analyzing the time-dependent statistics of the homodyne detector's output photocurrent with a continuous-wave LO. MacRae et al. ${ }^{16,17}$ showed that the autocorrelation function of this photocurrent estimates the real part of the density matrix defining the photon's temporal mode. Subsequently, this approach has been utilized for the 'Schrödinger cat' and two-photon Fock states. ${ }^{18}$

However, this method does not yield any information about the imaginary part of the photon's temporal density matrix (TDM). In this paper we present an experimental technique of polychromatic optical heterodyne tomography, which relies on acquiring the autocorrelation data of the homodyne photocurrent at multiple LO frequencies. The method enables us to determine both the real and imaginary parts of the photon's TDM, or, equivalently, both its amplitude and phase, thereby completely characterizing its temporal state. It works equally well for pure and mixed temporal modes.

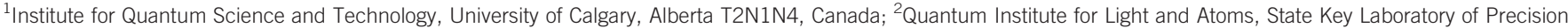

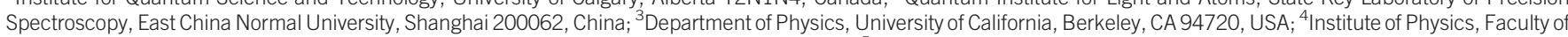

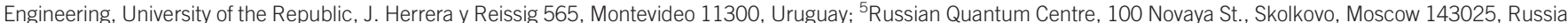
and ${ }^{6}$ Canadian Institute for Advanced Research, 180 Dundas Street West, Toronto, Ontario M5G 1Z8, Canada

Correspondence: Al Lvovsky, Institute for Quantum Science and Technology, University of Calgary, Alberta T2N1N4, Canada

E-mail: LVOV@ucalgary.ca

Received 26 September 2014; revised 20 February 2015; accepted 6 March 2015; accepted article preview online 7 March 2015 


\section{MATERIALS AND METHODS}

The (pure) temporal mode of a photon is defined by annihilation operator

$$
\hat{A}_{\phi}=\int_{-\infty}^{\infty} \hat{a}_{t} \phi(t) d t
$$

where $\phi(t)$ is the temporal mode function (TMF) and $\hat{a}_{t}$ represents the instantaneous annihilation operator at time $t$. Although single photons associated with a certain moment in time are ill-defined, treatment (1) is approximately valid as long as the spectral width of the photon is much less than its frequency. ${ }^{19} \mathrm{~A}$ single photon state in this mode is then given by $\left|1_{\phi}\right\rangle=\hat{A}_{\phi}^{\dagger}|0\rangle=\int_{-\infty}^{\infty} \phi^{*}(t)\left|1_{t}\right\rangle d t$, where $\left|1_{t}\right\rangle=\hat{a}_{t}^{\dagger}|0\rangle$.

The digital nature of the data acquisition system used in our experiment compels us to represent the temporal modes in terms of discrete time bins. The single photon state in temporal mode $\phi(t)$ can then be approximately expressed as $\left|1_{\phi}\right\rangle=\sum_{j} \phi^{*}\left(t_{j}\right)\left|1_{j}\right\rangle$ with $\sum_{j}\left|\phi\left(t_{j}\right)\right|^{2}=1$. Here $t_{j}$ is the time associated with the $j^{\text {th }}$ bin and $\left|1_{j}\right\rangle$ is the state containing one photon in the top-hat temporal mode associated with the $j^{\text {th }}$ bin and vacuum in all other bins. The density operator of the photon is then represented as $\sum_{m n} \rho_{m n}\left|1_{m}\right\rangle\left\langle 1_{n}\right|$ where $\rho_{m n}$ is the TDM.

The homodyne current for the $j^{\text {th }}$ time bin $I\left(t_{j}\right)$ is proportional to the quadrature

$$
\hat{X}_{j}=\left(\hat{a}_{j} e^{-i \theta_{j}}+\hat{a}_{j}^{\dagger} e^{i \theta_{j}}\right) / \sqrt{2}
$$

where $\theta_{j}=\delta \omega \cdot t_{j}+\theta_{0}$ is the optical phase difference between the LO and the signal. Here $\delta \omega$ is the frequency detuning between the LO and the signal and $\theta_{0}$ the LO relative phase at $t=0$. The autocorrelation matrix for the homodyne current is then

$$
\begin{array}{r}
\left\langle I\left(t_{j}\right) I\left(t_{k}\right)\right\rangle \propto\left\langle\hat{X}_{j} \hat{X}_{k}\right\rangle=\operatorname{Tr}\left[\hat{\rho} \hat{X}_{j} \hat{X}_{k}\right] \\
=\sum_{m n} \rho_{m n}\left\langle 1_{n}\left|\hat{X}_{j} \hat{X}_{k}\right| 1_{m}\right\rangle
\end{array}
$$

where each matrix element can be evaluated using Equation (2) as

$\left\langle 1_{n}\left|\hat{X}_{j} \hat{X}_{k}\right| 1_{m}\right\rangle=\frac{1}{2}\left[e^{-i \delta \omega\left(t_{k}-t_{j}\right)} \delta_{k m} \delta_{n j}+\delta_{j k} \delta_{n m}+e^{-i \delta \omega\left(t_{j}-t_{k}\right)} \delta_{j m} \delta_{n k}\right](4)$

Note that Equation (4) does not depend on $\theta_{0}$ due to the phase uncertainty of Fock states.

From Equations (3) and (4), one can obtain

$$
\left\langle\hat{X}_{j} \hat{X}_{k}\right\rangle=\frac{1}{2} \delta_{j k}+A_{j k}
$$

The first term in Equation (5) corresponds to the autocorrelation matrix for the vacuum. The second term, which we call the reduced autocorrelation matrix, is directly related to the photon's TDM:

$$
A_{j k}=\operatorname{Re}\left[\rho_{j k}\right] \cos \left[\delta \omega\left(t_{j}-t_{k}\right)\right]+\operatorname{Im}\left[\rho_{j k}\right] \sin \left[\delta \omega\left(t_{j}-t_{k}\right)\right]
$$

If the $\mathrm{LO}$ frequency is same as that of the signal, i.e., at $\delta \omega=0$, the autocorrelation matrix depends only on the real part of the TDM. However, by using $\delta \omega \neq 0$ one obtains access to its imaginary part.

In a realistic experiment, the photon being tested may experience losses, resulting in admixture of the vacuum into the state detected. Our technique would still apply to this case, but the second term would enter Equation (5) with the coefficient equal to the transmissivity of the lossy element. For high losses, acquisition of larger quantities of data may be necessary to reduce the statistical uncertainties (detailed further in the Supplementary Material).

Our experimental scheme for creating the single photon state and measuring the autocorrelation matrix is shown in Figure 1. We use coherent double Raman scattering (four-wave mixing) in an ensemble of $\Lambda$-type atoms to generate a two-mode squeezed state in a nondegenerate phase-matched configuration. ${ }^{17} \mathrm{~A}$ hot ${ }^{85} \mathrm{Rb}$ vapor cell is pumped by a 1 Watt laser beam at $795 \mathrm{~nm}$ derived from a continuouswave Ti:Sapphire laser. The signal and idler beams are spatially separated from the pump, and a specific spatial mode is selected in the idler channel using a single-mode fiber. Subsequently, the idler channel is subjected to spectral filtering by means of a lens cavity $(\mathrm{C} 1)$ of a $55 \mathrm{MHz}$ bandwidth ${ }^{20}$ and a conventional Fabry-Perot cavity (C2) of bandwidth $\gamma / 2 \pi=7 \mathrm{MHz}$. The usage of two cavities with incommensurate free spectral ranges ensures that the combined spectral filter has a single transmission peak of $7 \mathrm{MHz}$ width. This results in a heralded photon with a temporal mode that can be easily resolved by our homodyne detector with a $100 \mathrm{MHz}$ bandwidth. ${ }^{21}$

The idler beam is then coupled to a PerkinElmer single photon counting module (SPCM) with a dark count rate below $100 \mathrm{~Hz}$. Both cavities are maintained at a stable frequency by using an alignment beam which is unblocked every few seconds to monitor and readjust the cavity resonance frequency. Detection of an idler photon projects the signal onto a single photon in a well-defined spatiotemporal mode conjugate to the idler. This signal channel is modematched with a continuous-wave LO $(18 \mathrm{~mW})$ for homodyne detection. ${ }^{21}$ The LO is derived from a diode laser that is locked and phase stabilized with respect to the pump using an optical phase-lock loop. ${ }^{22}$

A click from the SPCM in the idler channel acts as the trigger for the measurement of the signal. The homodyne photocurrent is recorded for 360 ns around the trigger point as reference with a time binning of 2 ns. For each LO detuning, the autocorrelation matrix (3) of the homodyne photocurrent is obtained by taking an average over 2 million traces.

Theoretically, the data corresponding to two LO detunings would constitute a quorum for the temporal mode reconstruction. Experimentally, however, we take data at eight different detunings to avoid the situation where the sinusoids in Equation (6) approach zero for all detunings simultaneously and to enhance the statistical accuracy of the recovered $\hat{\rho}$.

Once the autocorrelation matrices have been acquired, we process them to eliminate the vacuum term in Equation (5), as well as any contributions from the DC (direct current) bias in the homodyne photocurrent and thermal background. These contributions are not correlated with trigger events, and are only dependent on the difference $t_{j}-t_{k}$. They can therefore be evaluated as the mean autocorrelation value along lines $t_{j}-t_{k}=$ const for the data points acquired significantly after the trigger pulse where no signal photon is expected. Subtracting them from the autocorrelation matrix yields the reduced autocorrelation matrix (Figure 2) (detailed further in the Supplementary Material).

The TDM can now be determined by solving Equation (6) for each pair $(j, k)$. However, such direct approach does not ensure positivity and normalization of the reconstructed density operator. To incorporate these a priori constraints into the reconstruction, we implement a more sophisticated iterative optimization algorithm. The algorithm uses the eight experimental reduced autocorrelation matrices as the training set. The difference between the experimental left-hand side of Equation (6) and the right-hand side of that equation evaluated from the estimated TDM, squared and summed over all pairs $(j, k)$ and 
a

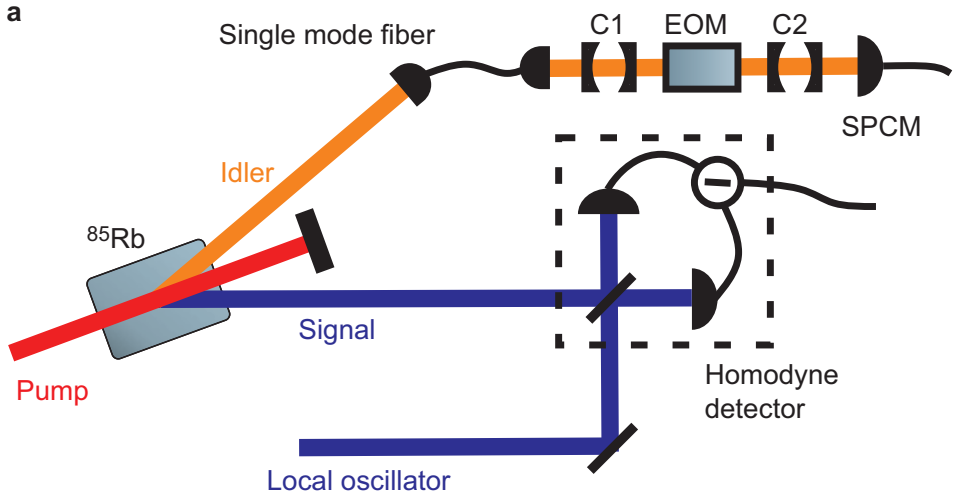

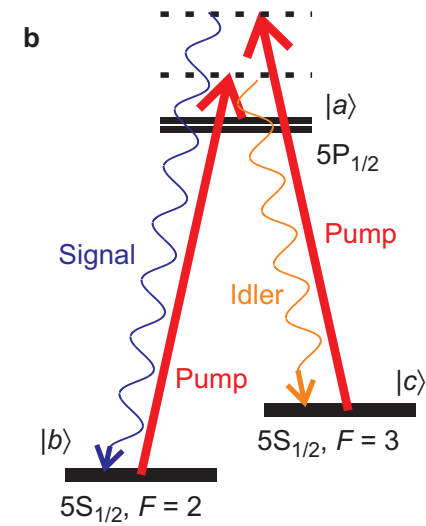

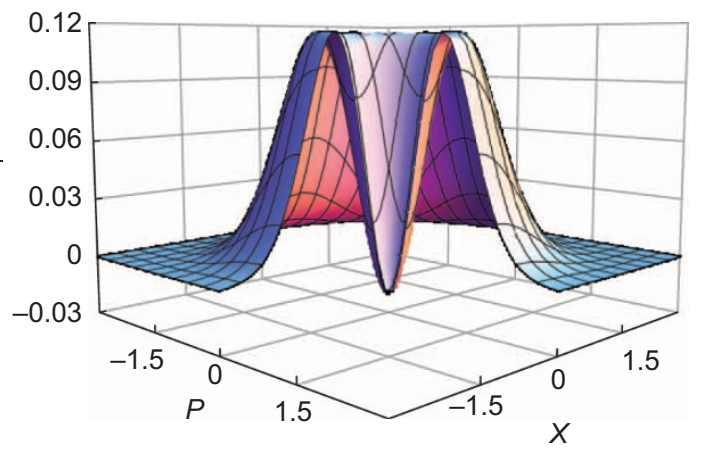

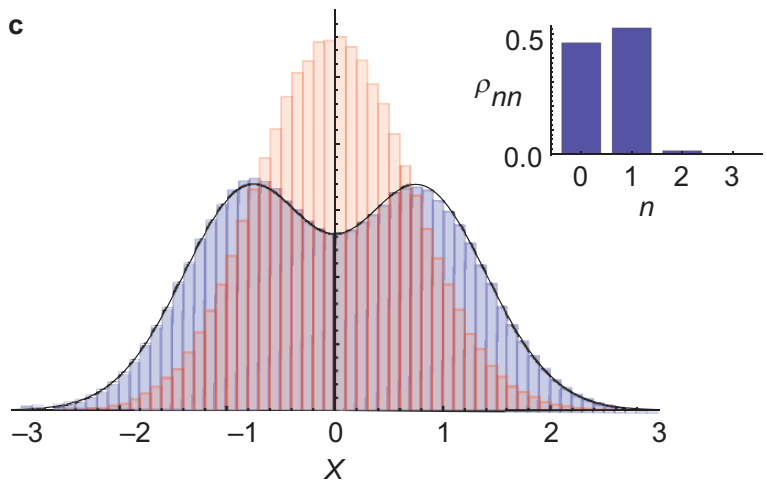

Figure 1. (a) Schematic of the experimental setup. The signal (blue) goes to the homodyne detector, whereas the idler (orange) passes through $\mathrm{C} 1$ ( $55 \mathrm{MHz}$ ) and $\mathrm{C} 2\left(7 \mathrm{MHz}\right.$ ) before detection via the SPCM. The EOM between $\mathrm{C} 1$ and $\mathrm{C} 2$ is optional. (b) The ${ }^{85} \mathrm{Rb}$ three-level $\Lambda$ system, with the fields' configuration shown. (c) Reconstruction of the state of the electromagnetic field in the temporal mode determined experimentally (unmodulated case). Left to right: experimental quadrature distribution (blue) overlaid with that for the vacuum state (red); diagonal elements of the Fock-basis density matrix; Wigner function. The single-photon fraction is 52.9\%. C1, C2: filter cavities; EOM: electro-optic modulator; SPCM: single photon counting module.

all LO frequencies, is used as the cost function. Iterations utilize the diagonal representation of the TDM: $\hat{\rho}=\sum_{i} p_{i}\left|\psi_{i}\right\rangle\left\langle\psi_{i}\right|$. In the first step of each iteration, the eigenvalues $p_{i}$ are adjusted to minimize the cost function while keeping them real, non-negative and totalling 1 . In the second step, the eigenvectors $\left|\psi_{i}\right\rangle$ are optimized by pairwise unitary transformations. The process is repeated until the cost function asymptotically converges to give the best fit of the TDM.

The theoretically expected mode is calculated from the properties of our experimental setup. The primary element determining the mode of the heralded photon is the narrowband filter cavity $\mathrm{C} 2$ in the idler channel. Additionally, the mode's bandwidth is limited by the $\sim 50 \mathrm{MHz}$ gain bandwidth of the four-wave mixing process used to generate the biphotons. This effect is taken into account in theoretical plots in Figures 2 and 3, however we neglect it in the theoretical expressions below for clarity.

The Lorentzian filter C2 in the idler channel produces a signal photon with the TMF in the shape of a rising exponential that terminates at the trigger event (detailed further in the Supplementary Material):

$$
\phi(t)=\sqrt{\gamma} e^{\gamma t / 2} \Theta(-t)
$$

where $\Theta(t)$ is the Heaviside step function and $\gamma=2 \pi \times 7 \mathrm{MHz}$ is the narrowband cavity linewidth. This exponentially rising mode is of particular significance for applications such as high-efficiency excitation of an atom ${ }^{23,24}$ or a resonator ${ }^{25,26}$ with a single photon.
To our knowledge, this is the first demonstration of complete reconstruction of this mode.

\section{RESULTS AND DISCUSSION}

Figure 3a shows the TDM obtained by iterative reconstruction from the experimental data along with the theoretical predictions. The primary eigenvector of the TDM has a corresponding eigenvalue almost 45 times larger than the second largest one indicating a nearly pure temporal mode. The TDM is primarily real and matches well the theoretical prediction.

Next, we demonstrate the reconstruction of a temporal mode with a nonvanishing imaginary component. To this end, we induce a virtual frequency shift by redefining the signal-LO detuning according to $\delta \omega^{\prime}$ $=\delta \omega+\Delta$ when reconstructing the TDM from Equation (6). The theoretically expected TMF and TDM then become:

$$
\begin{gathered}
\phi_{\Delta}(t)=\phi(t) e^{i \Delta t} \\
\rho_{\Delta}\left(t, t^{\prime}\right)=\rho\left(t, t^{\prime}\right) e^{i \Delta\left(t-t^{\prime}\right)}
\end{gathered}
$$

The TDM reconstructed from the experimental data using the effective modulation frequency of $\Delta=2 \pi \times 5 \mathrm{MHz}$ is shown in Figure $3 \mathrm{~b}$. While the purity of the temporal mode is maintained, the reconstructed density matrix now has a significant imaginary component, 

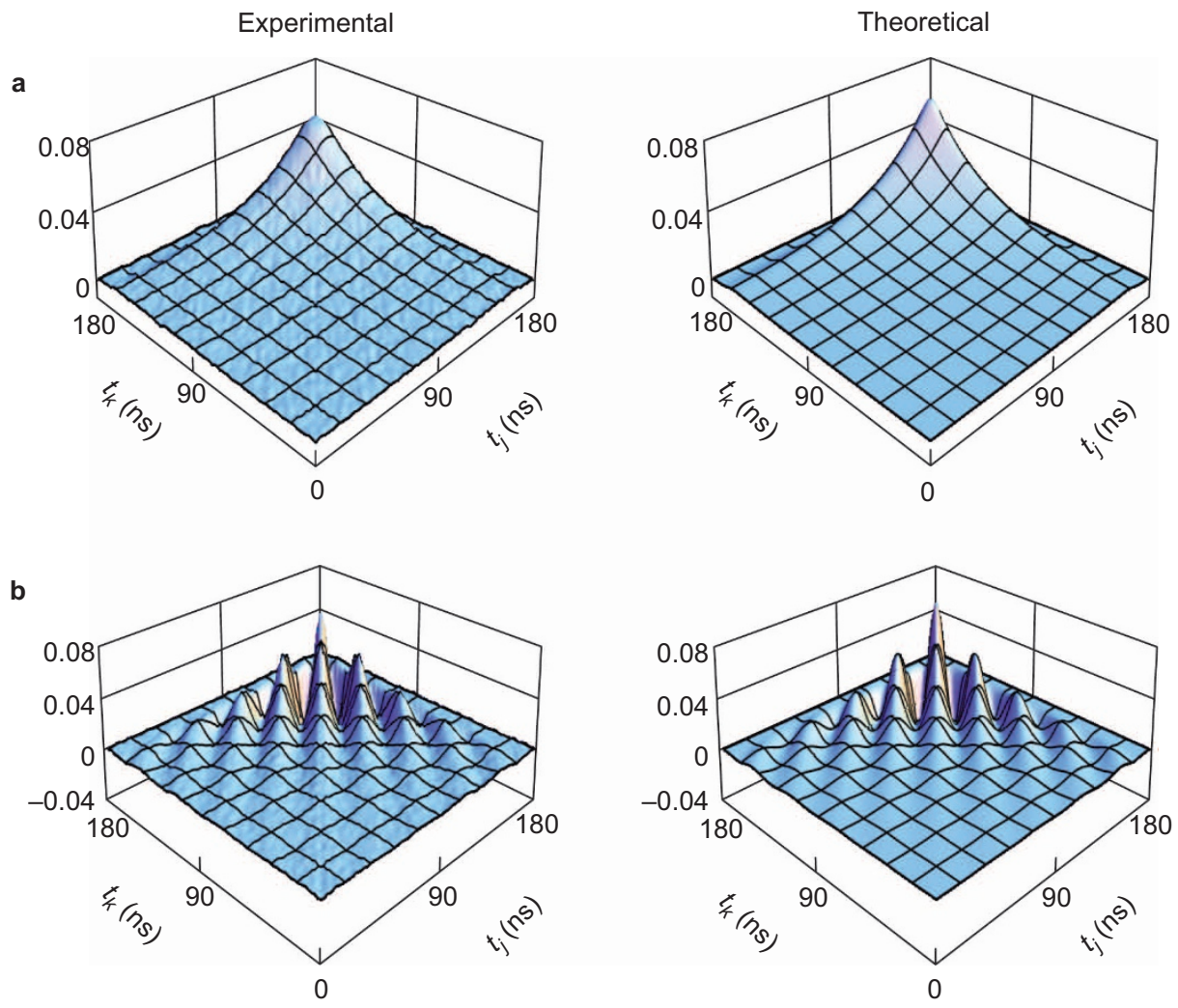

Figure 2. Theoretical (right) and experimental (left) reduced autocorrelation matrices, for two different local oscillator detunings: (a) $0 \mathrm{MHz}$ and (b) $27 \mathrm{MHz}$, corresponding to the measurement setting without modulation. The trigger photon arrives at $t=155 \mathrm{~ns}$.

demonstrating the ability of our technique to accurately reconstruct states with complex temporal modes.

This example is practically relevant in a situation when one does not know the frequency of the photon precisely. In this case, heterodyne measurements at different LO detunings relative to a given reference (defined by the point $\delta \omega=0$ ) will provide full information about the mode, including the spectral offset of the photon with respect to that reference.

Finally, we illustrate the ability of our experimental technique to reconstruct the TDM in the case of a mixed state. We phase modulate the signal photons at a frequency $\omega_{m}=2 \pi \times 20 \mathrm{MHz}$, larger than the spectral width of C2. This is achieved by passing the idler photons through an electro-optic modulator (EOM), with its optical axis oriented along the photon's polarization. ${ }^{27}$ This leads to a TMF $\phi^{\mathrm{EOM}}\left(t, \theta_{m}\right)=\sqrt{\gamma} e^{\gamma t / 2} e^{i \beta \sin \left(\omega_{m} t+\theta_{m}\right)} \Theta(-t)$, where $\beta=1.1$ is the modulation index and $\theta_{m}$ is the phase of the modulating voltage at the time when the idler photon is detected. Because the idler photon detections occur at random times, $\theta_{m}$ is randomized, leading to the following non-pure TDM:

$$
\begin{aligned}
& \rho_{t, t^{\prime}}^{\mathrm{EOM}}=\frac{1}{2 \pi} \int_{-\pi}^{\pi} \phi^{\mathrm{EOM}}\left(t, \theta_{m}\right)\left[\phi^{\mathrm{EOM}}\left(t^{\prime}, \theta_{m}\right)\right]^{*} d \theta_{m} \\
& =\gamma e^{\frac{\gamma\left(t+t^{\prime}\right)}{2}} \Theta(-t) \Theta\left(-t^{\prime}\right) J_{0}\left[2 \beta \sin \left(\frac{\omega_{m}\left(t-t^{\prime}\right)}{2}\right)\right]
\end{aligned}
$$

where $J_{0}$ is the Bessel function of the first kind.

The experimentally reconstructed TDM is shown in Figure 3c. The mixed nature of the density matrix is evident from the distribution of eigenvalues, with the ratio of the first and second eigenvalues being only around 2. Due to the modulation phase randomization, the imaginary part of the density matrix is zero.

The observed artifacts in the reconstructed photon modes can be attributed to the finite bandwidth, or non-instantaneous response, of the homodyne detector. This results in the smearing of the acquired autocorrelation matrix. This effect is particularly significant where this matrix has sharp features, such as the trigger event where the photon pulse instantly terminates according to Equation (7), as seen in Figure $3 \mathrm{a}$ and $3 \mathrm{~b}$. The fast modulation of the TMF such as in Figure $3 \mathrm{c}$ has a similar effect on the reconstructed mode over its entire duration, resulting in a spurious nonzero imaginary part. The observed artifacts, however, do not significantly degrade the fidelities of the experimentally obtained TDMs with respect to the theoretically expected ones. These fidelities, defined as $F=\operatorname{Tr}\left[\sqrt{\sqrt{\rho_{\exp }} \rho_{t h} \sqrt{\rho_{\exp }}}\right]$ with the subscripts indicating theory versus experiment, are found to be $0.97,0.94$, and 0.93 , respectively, for the three cases of Figure 3.

Using the absolute value of the TMF obtained for the primary mode of the unmodulated case (Figure 3a), we reconstruct the quantum state of light in that mode in the Fock basis akin to Ref.17, obtaining the single photon efficiency of $\rho_{11}=52.9 \%$. The corresponding Wigner function, exhibiting negative values at the phase-space origin, is plotted in Figure $1 \mathrm{c}$ along with the acquired quadrature distribution and the reconstructed density matrix.

\section{CONCLUSION}

We have developed and experimentally demonstrated polychromatic optical heterodyne tomography, a robust method for complete experimental determination of the temporal properties of a single photon directly from the time-resolved photocurrent statistics of a balanced 

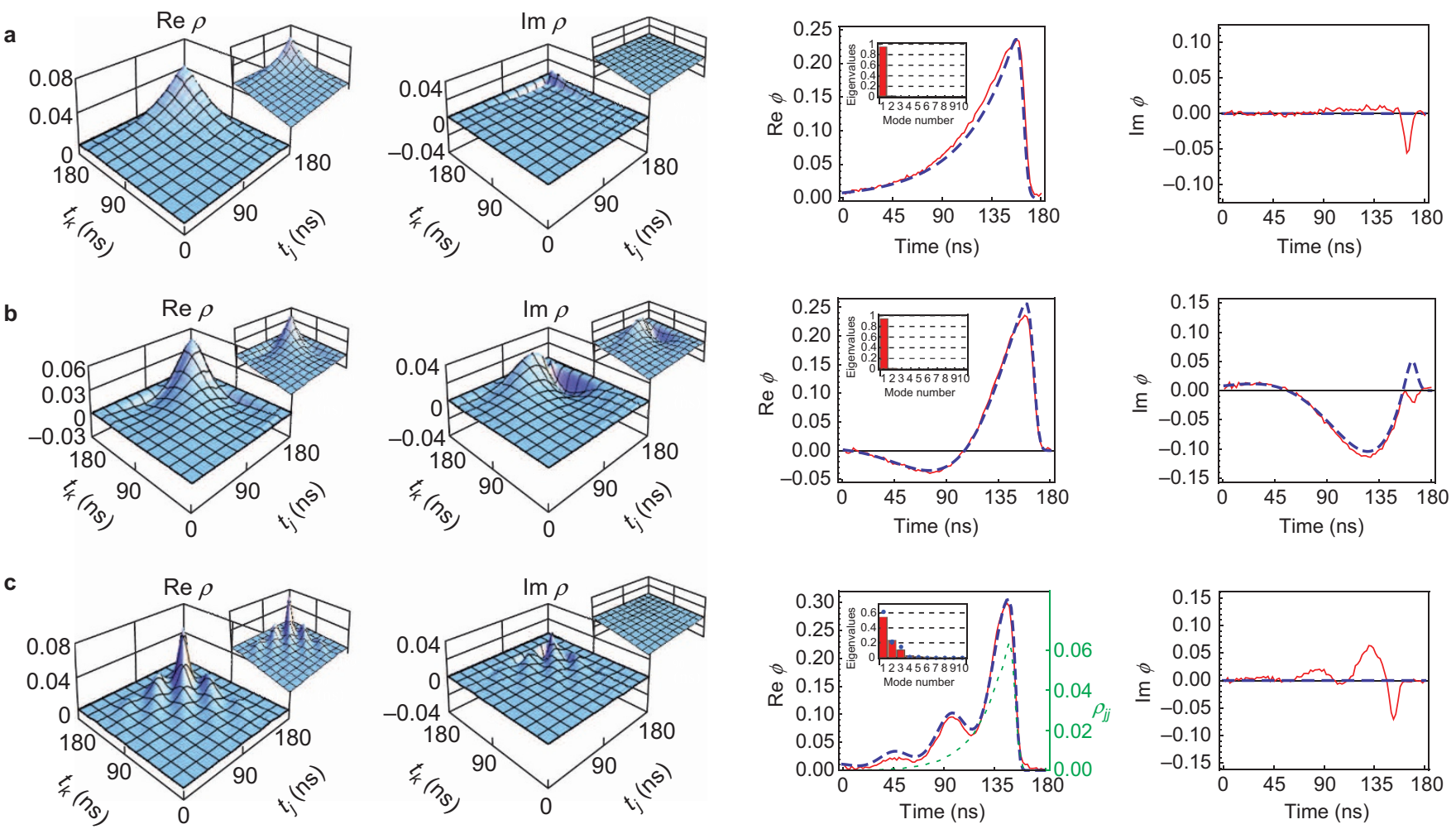

Figure 3. Experimentally reconstructed temporal modes and their theoretical predictions for the cases without modulation (a), with virtual phase modulation (b), and with phase-randomized EOM modulation (c). For each case, the first and second panels show the real (first) and imaginary (second) parts of the TDM, with the insets showing corresponding theoretical plots. The third and fourth panels show the TDM's primary eigenvector as reconstructed from experimental data (solid red) and theoretical modeling (dashed blue). The green dotted line in (c) also shows the diagonal of the TDM, which is not affected by the modulation as expected from Equation (10). The insets in the third panels show the distribution of eigenvalues (red bars) obtained experimentally. Without EOM modulation (a, b), the theoretically expected mode is pure so the TDM is expected to have only one nonvanishing eigenvector. In the case with EOM modulation (c), the TDM is mixed and the solid blue dots in the inset show theoretical eigenvalues. The trigger photon arrives at $t=155 \mathrm{~ns}$ for (a), (b) and at $t=145 \mathrm{~ns}$ for (c). EOM, electro-optic modulator; TDM, temporal density matrix.

homodyne measurement. The method enables the extraction of a temporal mode which in general may be complex and can have multiple frequency components. Accurate detection of the temporal mode is key for the proper mode matching required by many quantum communication protocols.

Our method permits straightforward extension to states other than the single photon Fock state akin to Ref.18 provided that the state in question occupies a well-defined spatiotemporal mode. On the other hand, the single photon state is special in that it can be directly associated with the photon annihilation operator of a certain optical mode or a mixture thereof. The problem of defining the optical mode(s) for a general quantum optical state is a subject of a separate study.

Although the technique described in this work requires the frequency spectrum of the photon's temporal mode to be sufficiently narrowband so its temporal structure can be resolved by the homodyne detector, one can envision ways to lift this restriction. For example, if the photon is produced in an ultrashort pulsed mode, one can extend it in time using a dispersive element such as an optical fiber, and perform time-resolved homodyne detection using a matched chirped LO. The time-domain correlations of the homodyne photocurrent will then correspond to quantum coherences between components of the photon spectrum.

\section{ACKNOWLEDGEMENTS}

ZQ and ASP contributed equally to this work. We thank Erhan Saglamyurek and Wolfgang Tittel for lending us the EOM. The project is supported by
NSERC and CIFAR. AL is a CIFAR Fellow. ZQ is supported by the China Scholarship Council.

1 Gisin N, Ribordy G, Tittel W, Zbinden H. Quantum cryptography. Rev Mod Phys 2002; 74: 145-195.

2 Bouwmeester D, Pan JW, Mattle K, Eibl M, Weinfurter $\mathrm{H}$ et al. Experimental quantum teleportation. Nature 1997; 390: 575-579.

3 Duan LM, Lukin MD, Cirac JI, Zoller P. Long-distance quantum communication with atomic ensembles and linear optics. Nature 2001; 414: 413-418.

4 Knill E, Laflamme R, Milburn GJ. A scheme for efficient quantum computation with linear optics. Nature 2001; 409: 46-52.

5 Grosshans F, Grangier P. Effective quantum efficiency in the pulsed homodyne detection of a n-photon state. Eur Phys J D 2001; 14: 119-125.

6 Aichele T, Lvovsky Al, Schiller S. Optical mode characterization of single photons prepared by means of conditional measurements on a biphoton state. Eur Phys J D 2002; 18: 237-245.

7 Keller M, Lange B, Hayasaka K, Lange W, Walther H. Continuous generation of single photons with controlled waveform in an ion-trap cavity system. Nature 2004; 431: 1075-1078.

8 Mølmer K. Non-Gaussian states from continuous-wave Gaussian light sources. Phys Rev A 2006; 73: 063804.

9 Sasaki M, Suzuki S. Multimode theory of measurement-induced non-Gaussian operation on wideband squeezed light: analytical formula. Phys Rev A 2006; 73: 043807.

10 Lvovsky Al, Raymer MG. Continuous-variable optical quantum-state tomography. Rev Mod Phys 2009; 81: 299-332.

11 Lundeen JS, Sutherland B, Patel A, Stewart C, Bamber C. Direct measurement of the quantum wavefunction. Nature 2011; 474: 188-191.

12 Polyakov SV, Chou CW, Felinto D, Kimble HJ. Temporal dynamics of photon pairs generated by an atomic ensemble. Phys Rev Lett 2004; 93: 263601.

13 Flagg EB, Polyakov SV, Thomay T, Solomon GS. Dynamics of nonclassical light from a single solid-state quantum emitter. Phys Rev Lett 2012; 109: 163601 
14 Polycarpou C, Cassemiro KN, Venturi G, Zavatta A, Bellini M. Adaptive detection of arbitrarily shaped ultrashort quantum light states. Phys Rev Lett2012; 109: 053602.

15 Wasilewski W, Kolenderski P. Frankowski R. Spectral density matrix of a single photon measured. Phys Rev Lett 2007; 99: 123601.

16 MacRae A. An atomic source of quantum light. PhD thesis, University of Calgary, Calgary, 2012.

17 MacRae A, Brannan T, Achal R, Lvovsky AI. Tomography of a high-purity narrowband photon from a transient atomic collective excitation. Phys Rev Lett 2012; 109: 033601.

18 Morin O, Fabre C, Laurat J. Experimentally accessing the optimal temporal mode of traveling quantum light states. Phys Rev Lett 2013; 111: 213602.

19 Fedorov MV, Efremov MA, Kazakov AE, Chan KW, Law CK et al. Spontaneous emission of a photon: wave-packet structures and atom-photon entanglement. Phys Rev $A$ 2005; 72: 032110.

20 Palittapongarnpim P, MacRae A, Lvovsky Al. Note: A monolithic filter cavity for experiments in quantum optics. Rev Sci Instrum 2012; 83: 066101.

21 Kumar R, Barrios E, MacRae A, Cairns E, Huntington EH et al. Versatile wideband balanced detector for quantum optical homodyne tomography. Opt Comm 2012; 285: 5259-5267.

22 Appel J, MacRae A, Lvovsky AI. A versatile digital GHz phase lock for external cavity diode lasers. Meas Sci Technol 2009; 20: 055302.
23 Aljunid SA, Maslennikov G, Wang YM, Lan DH, Scarani V et al. Excitation of a single atom with exponentially rising light pulses. Phys Rev Lett 2013; 111: 103001.

24 Gulati GK, Srivathsan B, Chng B, Cerè A, Matsukevich D et al. Generation of an exponentially rising single-photon field from parametric conversion in atoms. Phys $\operatorname{Rev}$ A 2014; 90: 033819.

25 Bader M, Heugel S, Chekhov AL, Sondermann M, Leuchs G. Efficient coupling to an optical resonator by exploiting time-reversal symmetry. New J Phys 2013; 15 . 123008.

26 Liu C, Sun Y, Zhao LW, Zhang SC, Loy MMT et al. Efficiently loading a single photon into a single-sided Fabry-Perot cavity. Phys Rev Lett 2014; 113: 133601.

27 Kolchin P, Belthangady C, Du SW, Yin GY, Harris SE. Electro-optic modulation of single photons. Phys Rev Lett 2008; 101: 103601.

\section{(c) (i) $\Theta \Theta$ This work is licensed under a Creative Commons Attribution-}

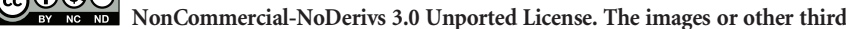
party material in this article are included in the article's Creative Commons license, unless indicated otherwise in the credit line; if the material is not included under the Creative

Commons license, users will need to obtain permission from the license holder to reproduce the material. To view a copy of this license, visit http://creativecommons.org/licenses/ by-nc-nd/3.0/

Supplementary Material for this article can be found on the Light: Science \& Applications' website (http://www.nature.com/lsa). 